\title{
An Introduction to Noncommutative Geometry
}

\author{
Joseph C. Várilly \\ Universidad de Costa Rica, \\ 2060 San José, Costa Rica
}

28 April 2006

\begin{abstract}
The lecture notes of this course at the EMS Summer School on Noncommutative Geometry and Applications in September, 1997 are now published by the EMS. Here are the contents, preface and updated bibliography from the published book.
\end{abstract}

\section{Contents}

1 Commutative Geometry from the Noncommutative Point of View

1.1 The Gelfand-Naumark cofunctors

1.2 The $\Gamma$ functor

1.3 Hermitian metrics and $\operatorname{spin}^{c}$ structures

1.4 The Dirac operator and the distance formula

2 Spectral Triples on the Riemann Sphere

2.1 Line bundles and the spinor bundle

2.2 The Dirac operator on the sphere $\mathbb{S}^{2}$

2.3 Spinor harmonics and the spectrum of $\not D$

2.4 Twisted spinor modules

2.5 A reducible spectral triple

3 Real Spectral Triples: the Axiomatic Foundation

3.1 The data set

3.2 Infinitesimals and dimension

3.3 The first-order condition

3.4 Smoothness of the algebra

3.5 Hochschild cycles and orientation

3.6 Finiteness of the $K$-cycle 
3.7 Poincaré duality and $K$-theory

3.8 The real structure

\section{Geometries on the Noncommutative Torus}

4.1 Algebras of Weyl operators

4.2 The algebra of the noncommutative torus

4.3 The skeleton of the noncommutative torus

4.4 A family of spin geometries on the torus

\section{The Noncommutative Integral}

5.1 The Dixmier trace on infinitesimals

5.2 Pseudodifferential operators

5.3 The Wodzicki residue

5.4 The trace theorem

5.5 Integrals and zeta residues

\section{Quantization and the Tangent Groupoid}

6.1 Moyal quantizers and the Moyal deformation

6.2 Smooth groupoids

6.3 The tangent groupoid

6.4 Moyal quantization as a continuity condition

6.5 The hexagon and the analytical index

6.6 Quantization and the index theorem

\section{Equivalence of Geometries}

7.1 Unitary equivalence of spin geometries

7.2 Morita equivalence and connections

7.3 Vector bundles over noncommutative tori

7.4 Morita-equivalent toral geometries

7.5 Gauge potentials

\section{Action Functionals}

8.1 Algebra automorphisms and the metric

8.2 The fermionic action

8.3 The spectral action principle

8.4 Spectral densities and asymptotics

\section{Epilogue: New Directions}

9.1 Noncommutative field theories

9.2 Isospectral deformations

9.3 Geometries with quantum group symmetry

9.4 Other developments 


\section{Introduction}

This book consists of lecture notes for a course given at the EMS Summer School on Noncommutative Geometry and Applications, at Monsaraz and Lisboa, Portugal in September, 1997. These were made available in preprint form on the ArXiv, as physics/9709045, at that time. In updating them for publication, I have kept to the original plan, but have added citations of more recent papers throughout. An extra final chapter summarizes some of the developments in noncommutative geometry in the intervening years.

The course sought to address a mixed audience of students and young researchers, both mathematicians and physicists, and to provide a gateway to noncommutative geometry, as it then stood. It already occupied a wide-ranging area of mathematics, and had received some scrutiny from particle physicists. Shortly thereafter, links to string theory were found, and its interest for theoretical physicists is now indisputable.

Many approaches can be taken to introducing noncommutative geometry. In these lectures, the focus is on the geometry of Riemannian spin manifolds and their noncommutative cousins, which are 'spectral triples' determined by a suitable generalization of the Dirac operator. These 'spin geometries', which are spectral triples with certain extra properties, underlie the noncommutative geometry approach to phenomenological particle models and recent attempts to place gravity and matter fields on the same geometrical footing.

The first two chapters are devoted to commutative geometry; we set up the general framework and then compute a simple example, the two-sphere, in noncommutative terms. The general definition of a spin geometry is then laid out and exemplified with the noncommutative torus. Enough details are given so that one can see clearly that noncommutative geometry is just ordinary geometry, extended by discarding the commutativity assumption on the coordinate algebra. Classification up to equivalence is dealt with briefly in Chapter 7 .

Other chapters explore some of the tools of the trade: the noncommutative integral, the role of quantization, and the spectral action functional. Physical models are not treated directly (these were the subject of other lectures at the Summer School), but most of the mathematical issues needed for their understanding are dealt with here. The final chapter is a brief overview of the profusion of new examples and applications of noncommutative spaces and spectral triples.

I wish to thank several people who contributed in no small way to assembling these lecture notes. José M. Gracia-Bondía gave decisive help at many points; and Alejandro Rivero provided constructive criticism. I thank Daniel Kastler, Bruno Iochum, Thomas Schücker and the late Daniel Testard for the opportunity to visit the Centre de Physique Thorique of the CNRS at Marseille, as a prelude to the Summer School; and Piotr M. Hajac for an invitation to teach at the University of Warsaw, when I rewrote the notes for publication. This visit to Katedra Metod Matematycznych Fizyki of UW was supported by European Commission grant MKTD-CT-2004-509794.

I am grateful for enlightening discussions with Alain Connes, Robert Coquereaux, Ricardo Estrada, Héctor Figueroa, Thomas Krajewski, Giovanni Landi, Fedele Lizzi, Carmelo Pérez Martín, William J. Ugalde and Mark Villarino. Thanks also to Jesús Clemente, Stephan de Bièvre and Markus Walze who provided indispensable references. Several improvements 
to the original draft notes were suggested by Eli Hawkins, Thomas Schücker and Georges Skandalis. Last but by no means least, I want to discharge a particular debt of gratitude to Paulo Almeida for his energy and foresight in organizing the Summer School in the right place at the right time.

San Pedro de Montes de Oca

April 2006

\section{The Lectures}

The main body of the book is a revised and updated version of the lectures. A final chapter, reviewing developments up to 2005, has been added. The book is now published by the European Mathematical Society, Zürich, 2006, as the fourth volume in the EMS Series of Lectures in Mathematics.

\section{References}

[1] J. Aastrup, R. Nest and E. Schrohe, A continuous field of $C^{*}$-algebras and the tangent groupoid for manifolds with boundary. J. Funct. Anal. (2006), to appear.

[2] E. Álvarez, J. M. Gracia-Bondía and C. P. Martín, Parameter constraints in a noncommutative geometry model do not survive standard quantum corrections. Phys. Lett. B 306 (1993), 55-58.

[3] E. Álvarez, J. M. Gracia-Bondía and C. P. Martín, A renormalization group analysis of the NCG constraints $m_{\mathrm{top}}=2 m_{W}, m_{\mathrm{Higgs}}=3.14 m_{W}$. Phys. Lett. B 329 (1994), 259-262.

[4] M. F. Atiyah, R. Bott and A. Shapiro, Clifford Modules. Topology 3 (1964), 3-38.

[5] M. F. Atiyah and I. M. Singer, The index of elliptic operators I. Ann. of Math. 87 (1968), 484-530.

[6] I. Avramidi, Heat kernel approach in quantum field theory. Nuclear Phys. B (Proc. Suppl.) 104 (2002), 3-32.

[7] P. Baum, P. M. Hajac, R. Matthes and W. Szymański, Noncommutative geometry approach to principal and associated bundles. In Quantum Symmetry and Noncommutative Geometry (ed. by P. M. Hajac), forthcoming, 2006.

[8] F. Bayen, M. Flato, C. Fronsdal, A. Lichnerowicz and D. Sternheimer, Deformation theory and quantization. Ann. Physics (NY) 111 (1978), 61-110 and 111-151.

[9] J. Bellissard, A. van Elst and H. Schulz-Baldes, The noncommutative geometry of the quantum Hall effect. J. Math. Phys. 35 (1994), 5373-5451. 
[10] M.-T. Benameur and T. Fack, Type II noncommutative geometry. I. Dixmier trace in von Neumann algebras. Adv. Math. 199 (2006), 29-87.

[11] F. A. Berezin, The Method of Second Quantization. Academic Press, New York, 1966.

[12] N. Berline, E. Getzler and M. Vergne, Heat Kernels and Dirac Operators. Grundlehren Math. Wiss. 298, Springer-Verlag, Berlin, 1992.

[13] B. Blackadar, K-theory for Operator Algebras. Second edition, Math. Sci. Res. Inst. Publ. 5, Cambridge University Press, Cambridge, 1998.

[14] D. Borthwick and A. Uribe, Almost complex structures and geometric quantization. Math. Res. Lett. 3 (1996), 845-861.

[15] J.-B. Bost, Principe d'Oka, $K$-théorie et systèmes dynamiques non commutatifs. Invent. Math. 101 (1990), 261-333.

[16] O. Bratteli, G. A. Elliott and P. E. T. Jørgensen, Decomposition of unbounded derivations into invariant and approximately inner parts. J. Reine Angew. Math. 346 (1984), 166-193.

[17] J. Brodzki, An Introduction to K-theory and Cyclic Cohomology. Polish Scientific Publishers (PWN), Warszawa, 1998.

[18] R. Brout, Notes on Connes' construction of the Standard Model. Nuclear Phys. B (Proc. Suppl.) 65 (1998), 3-15.

[19] L. G. Brown, P. Green and M. A. Rieffel, Stable isomorphism and strong Morita equivalence of $C^{*}$-algebras. Pacific. J. Math. 71 (1977), 349-363.

[20] A. L. Carey, J. Phillips, A. Rennie and F. A. Sukochev, The Hochschild class of the Chern character for semifinite spectral triples. J. Funct. Anal. 213 (2004), 111-153.

[21] A. L. Carey, J. Phillips, A. Rennie and F. A. Sukochev, The local index formula in semifinite von Neumann algebras. I: Spectral flow. Adv. Math. 202 (2006), 451-516; II: The even case. Adv. Math. 202 (2006), 517-554.

[22] J. F. Cariñena, J. Clemente-Gallardo, E. Follana, J. M. Gracia-Bondía, A. Rivero and J. C. Várilly, Connes' tangent groupoid and strict quantization. J. Geom. Phys. 32 (1999), 79-96.

[23] J. F. Cariñena, J. M. Gracia-Bondía and J. C. Várilly, Relativistic quantum kinematics in the Moyal representation. J. Phys. A 23 (1990), 901-933.

[24] L. Carminati, B. Iochum and T. Schücker, The noncommutative constraints on the Standard Model à la Connes. J. Math. Phys. 38 (1997), 1269-1280. 
[25] P. S. Chakraborty and A. Pal, Equivariant spectral triples on the quantum SU(2) group. K-Theory 28 (2003), 107-126.

[26] A. H. Chamseddine and A. Connes, The spectral action principle. Commun. Math. Phys. 186 (1997), 731-750.

[27] A. H. Chamseddine and A. Connes, Scale invariance in the spectral action. Preprint hep-th/0512169, Beirut, 2005.

[28] I. Chepelev and R. Roiban, Renormalization of quantum field theories on noncommutative $\mathbb{R}^{d}$, I: Scalars. J. High Energy Phys. 0005 (2000), 037.

[29] I. Chepelev and R. Roiban, Convergence theorem for noncommutative Feynman graphs and renormalization. J. High Energy Phys. 0103 (2001), 001.

[30] E. Christensen and C. Ivan, Sums of two dimensional spectral triples. Preprint math.oa/0601024, Copenhagen, 2006.

[31] F. Cipriani, D. Guido and S. Scarlatti, A remark on trace properties of $K$-cycles. $J$. Operator Theory 35 (1996), 179-189.

[32] A. Connes, $C^{*}$-algèbres et géométrie différentielle. C. R. Acad. Sci. Paris 290A (1980), 599-604. English translation: $C^{*}$-algebras and differential geometry. In Physics in Noncommutative World I: Field Theories (ed. by M. Li and Y.-S. Wu), Rinton Press, Paramus, NJ, 2002, 39-45.

[33] A. Connes, A survey of foliations and operator algebras. In Operator Algebras and Applications (ed. by R. V. Kadison), Proc. Sympos. Pure Math. 38, Amer. Math. Soc., Providence, RI, 1982, 521-628.

[34] A. Connes, Noncommutative differential geometry. Inst. Hautes tudes Sci. Publ. Math. 62 (1985), 257-360.

[35] A. Connes, The action functional in noncommutative geometry. Commun. Math. Phys. 117 (1988), 673-683.

[36] A. Connes, Compact metric spaces, Fredholm modules, and hyperfiniteness. Ergodic Theory Dynam. Systems 9 (1989), 207-220.

[37] A. Connes, Noncommutative Geometry. Academic Press, London, 1994.

[38] A. Connes, Geometry from the spectral point of view. Lett. Math. Phys. 34 (1995), 203-238.

[39] A. Connes, Noncommutative geometry and reality. J. Math. Phys. 36 (1995), 61946231. 
[40] A. Connes, Gravity coupled with matter and foundation of noncommutative geometry. Commun. Math. Phys. 182 (1996), 155-176.

[41] A. Connes, Brisure de symétrie spontanée et géométrie du point de vue spectral. Séminaire Bourbaki, 48ème année, exposé 816; J. Geom. Phys. 23 (1997), 206-234.

[42] A. Connes, A short survey of noncommutative geometry. J. Math. Phys. 41 (2000), $3832-3866$.

[43] A. Connes, Cyclic cohomology, noncommutative geometry and quantum group symmetries. In Noncommutative Geometry (ed. by S. Doplicher and R. Longo). Lecture Notes in Math. 1831, Springer-Verlag, Berlin, 2004, 1-71.

[44] A. Connes, C. Consani and M. Marcolli, Noncommutative geometry and motives: the thermodynamics of endomotives. Preprint math.qa/0512138, IHES, Bures-sur-Yvette, 2005.

[45] A. Connes, M. R. Douglas and A. Schwartz, Noncommutative geometry and Matrix theory: compactification on tori. J. High Energy Phys. 9802 (1998), 003.

[46] A. Connes and M. Dubois-Violette, Noncommutative finite-dimensional manifolds. I. Spherical manifolds and related examples. Commun. Math. Phys. 230 (2002), 539-579.

[47] A. Connes and M. Dubois-Violette, Noncommutative finite-dimensional manifolds. II. Moduli space and structure of noncommutative 3-spheres. Preprint math.qa/0511337, IHES, Bures-sur-Yvette, 2005.

[48] A. Connes and N. Higson, Déformations, morphismes asymptotiques et $K$-théorie bivariante. C. R. Acad. Sci. Paris 311 (1990), 101-106.

[49] A. Connes and G. Landi, Noncommutative manifolds, the instanton algebra and isospectral deformations. Commun. Math. Phys. 221 (2001), 141-159.

[50] A. Connes and J. Lott, Particle models and noncommutative geometry. Nuclear Phys. $B$ (Proc. Suppl.) 18 (1990), 29-47.

[51] A. Connes and M. Marcolli, A walk in the noncommutative garden. Preprint math.qa/0601054, IHES, Bures-sur-Yvette, 2006.

[52] A. Connes and H. Moscovici, The local index theorem in noncommutative geometry. Geom. Funct. Anal. 5 (1995), 174-243.

[53] A. Connes and H. Moscovici, Hopf algebras, cyclic cohomology and the transverse index theorem. Commun. Math. Phys. 198 (1998), 198-246.

[54] A. Connes and H. Moscovici, Background independent geometry and Hopf cyclic cohomology. In Quantum Symmetry and Noncommutative Geometry (ed. by P. M. Hajac), forthcoming, 2006. 
[55] A. Connes and M. A. Rieffel, Yang-Mills for noncommutative two-tori. In Operator Algebras and Mathematical Physics (ed. by E. T. Palle Jrgensen and Paul S. Muhly), Contemp. Math. 62, Amer. Math. Soc., Providence, RI, 1987, 237-266.

[56] R. Coquereaux, On the finite dimensional quantum group $M_{3} \oplus\left(M_{2 \mid 1}\left(\Lambda^{2}\right)\right)_{0}$. Lett. Math. Phys. 42 (1997), 309-328.

[57] J. Cuntz, Cyclic theory, bivariant $K$-theory and the bivariant Chern-Connes character. In Cyclic Homology in Noncommutative Geometry (ed. by J. Cuntz, G. Skandalis and B. Tsygan), Encyclopaedia Math. Sci. 121. Springer-Verlag, Berlin, 2004, 1-71.

[58] J. Cuntz and D. Quillen, Algebra extensions and nonsingularity. J. Amer. Math. Soc. 8 (1995), 251-289.

[59] L. Dąbrowski, G. Landi, M. Paschke and A. Sitarz, The spectral geometry of the equatorial Podleś sphere. C. R. Acad. Sci. Paris 340 (2005), 819-822.

[60] L. Dą̧browski, G. Landi, A. Sitarz, W. D. van Suijlekom and J. C. Várilly, The Dirac operator on $\mathrm{SU}_{q}(2)$. Commun. Math. Phys. 259 (2005), 729-759.

[61] L. Dąbrowski, F. Nesti and P. Siniscalco, A finite quantum symmetry of $M(3, \mathbb{C})$. Int. J. Mod. Phys. A 13 (1998), 4147-4162.

[62] L. Dąbrowski and A. Sitarz, Dirac operator on the standard Podleś quantum sphere. In Noncommutative Geometry and Quantum Groups (ed. by P. M. Hajac and W. Pusz), Banach Center Publications 61, PWN, Warsaw, 2003, 49-58.

[63] S. De Bièvre, Chaos, quantization and the classical limit on the torus. In Quantization, Coherent States, and Poisson Structures (ed. by A. Strasburger et al.), PWN, Warsaw, 1998, 19-39.

[64] J. A. Dieudonné, Treatise on Analysis. Vol. 3, Academic Press, New York, 1972.

[65] J. A. Dieudonné, Éléments d'Analyse. Vol. 9, Gauthier-Villars, Paris, 1982.

[66] J. Dixmier, Existence de traces non normales. C. R. Acad. Sci. Paris 262A (1966), $1107-1108$.

[67] J. Dixmier, Les $C^{*}$-algèbres et leurs Représentations. Gauthier-Villars, Paris, 1969.

[68] S. Doplicher, K. Fredenhagen and J. E. Roberts, The quantum structure of spacetime at the Planck scale and quantum fields. Commun. Math. Phys. 172 (1995), 187-220.

[69] M. R. Douglas and C. M. Hull, D-branes and the noncommutative torus. J. High Energy Phys. 9802 (1998), 008.

[70] M. R. Douglas and N. A. Nekrasov, Noncommutative field theory. Rev. Mod. Phys. 73 (2002), 977-1029. 
[71] M. Dubois-Violette, R. Kerner and J. Madore, Gauge bosons in a noncommutative geometry. Phys. Lett. B 217 (1989), 485-488.

[72] E. Elizalde, L. Vanzo and S. Zerbini, Zeta-function regularization, the multiplicative anomaly and the Wodzicki residue. Commun. Math. Phys. 194 (1998), 613-630.

[73] G. A. Elliott, On the $K$-theory of the $C^{*}$-algebra generated by a projective representation of a torsion-free discrete abelian group. In Operator Algebras and Group Representations 1 (ed. by G. Arsene), Pitman, London, 1984, 157-184.

[74] G. A. Elliott and H. Li, Morita equivalence of smooth noncommutative tori. Preprint math.oa/0311502, Toronto, 2003.

[75] G. A. Elliott and H. Li, Strong Morita equivalence of higher-dimensional noncommutative tori II. Preprint math.oa/0501030, Toronto, 2005.

[76] G. A. Elliott, T. Natsume and R. Nest, The Atiyah-Singer index theorem as passage to the classical limit in quantum mechanics. Commun. Math. Phys. 182 (1996), 505-533.

[77] G. G. Emch, Chaotic dynamics in noncommutative geometry. In Quantization, Coherent States, and Poisson Structures (ed. by A. Strasburger et al.), PWN, Warsaw, 1998, 193-205.

[78] R. Estrada, The Cesàro behaviour of distributions. Proc. Roy. Soc. London A 454 (1998), 2425-2443.

[79] R. Estrada and S. A. Fulling, Distributional asymptotic expansions of spectral functions and of the associated Green kernels. Electron. J. Differential Equations 1999 (1999), 1-37.

[80] R. Estrada, J. M. Gracia-Bondía and J. C. Várilly, On summability of distributions and spectral geometry. Commun. Math. Phys. 191 (1998), 219-248.

[81] R. Estrada and R. P. Kanwal, A Distributional Approach to Asymptotics: Theory and Applications. Birkhuser Advanced Texts, Birkhäuser, Boston, 2002.

[82] T. Fack and H. Kosaki, Generalized s-numbers of $\tau$-measurable operators. Pacific. J. Math. 123 (1986), 269-300.

[83] B. V. Fedosov, Deformation Quantization and Index Theory. Akademie Verlag, Berlin, 1996.

[84] B. V. Fedosov, F. Golse, E. Leichtman and E. Schrohe, The noncommutative residue for manifolds with boundary. J. Funct. Anal. 142 (1996), 1-31.

[85] H. Figueroa, F. Lizzi, J. M. Gracia-Bondía and J. C. Várilly, A nonperturbative form of the spectral action principle in noncommutative geometry. J. Geom. Phys. 26 (1998), 329-339. 
[86] T. Filk, Divergences in a field theory on quantum space, Phys. Lett. B 376 (1996), $53-58$.

[87] G. B. Folland, Harmonic analysis of the de Rham complex on the sphere. J. Reine Angew. Math. 398 (1989), 130-143.

[88] D. S. Freed, Review of 'The heat kernel Lefschetz fixed point formula for the $\mathrm{Spin}^{c}$ Dirac operator' by J. J. Duistermaat. Bull. Amer. Math. Soc. 34 (1997), 73-78.

[89] T. Friedrich, Dirac Operators in Riemannian Geometry. Grad. Stud. in Math. 25, Amer. Math. Soc., Providence, RI, 2000.

[90] J. Fröhlich, O. Grandjean and A. Recknagel, Supersymmetric quantum theory and (noncommutative) differential geometry. Commun. Math. Phys. 193 (1998), 527-594.

[91] V. Gayral, J. M. Gracia-Bondía, B. Iochum, T. Schücker and J. C. Várilly, Moyal planes are spectral triples. Commun. Math. Phys. 246 (2004), 569-623.

[92] V. Gayral, J. M. Gracia-Bondía and F. Ruiz-Ruiz, Trouble with space-like noncommutative field theory. Phys. Lett. B 610 (2005), 141-146.

[93] V. Gayral, B. Iochum and J. C. Várilly, Dixmier traces on noncompact isospectral deformations. Preprint hep-th/0507206. J. Funct. Anal. (2006), to appear.

[94] I. M. Gelfand and M. A. Naĭmark, On the embedding of normed rings into the ring of operators in Hilbert space. Mat. Sbornik 12 (1943), 197-213.

[95] I. M. Gelfand and N. Ya. Vilenkin, Generalized Functions 4: Applications of Harmonic Analysis. Academic Press, New York, 1964.

[96] P. B. Gilkey, Invariance Theory, the Heat Equation, and the Atiyah-Singer Index Theorem. CRC Press, Boca Raton, FL, 1995.

[97] J. N. Goldberg, A. J. Macfarlane, E. T. Newman, F. Rohrlich and E. C. G. Sudarshan, Spin-s spherical harmonics and ð. J. Math. Phys. 8 (1967), 2155-2161.

[98] J. Gomis and T. Mehen, Space-time noncommutative field theories and unitarity. Nuclear Phys. B 591 (2000), 265-270.

[99] J. M. Gracia-Bondía, Generalized Moyal quantization on homogeneous symplectic spaces. In Deformation Theory and Quantum Groups with Application to Mathematical Physics (ed. by J. Stasheff and M. Gerstenhaber), Contemp. Math. 134, Amer. Math. Soc., Providence, RI, 1992, 93-114.

[100] J. M. Gracia-Bondía, B. Iochum and T. Schücker, The Standard Model in noncommutative geometry and fermion doubling. Phys. Lett. B 416 (1998), 123-128. 
[101] J. M. Gracia-Bondía, F. Lizzi, G. Marmo and P. Vitale, Infinitely many star-products to play with. J. High Energy Phys. 0204 (2002) 026.

[102] J. M. Gracia-Bondía and J. C. Várilly, Algebras of distributions suitable for phasespace quantum mechanics I. J. Math. Phys. 29 (1988), 869-879.

[103] J. M. Gracia-Bondía and J. C. Várilly, ¿From geometric quantization to Moyal quantization. J. Math. Phys. 36 (1995), 2691-2701.

[104] J. M. Gracia-Bondía, J. C. Várilly and H. Figueroa, Elements of Noncommutative Geometry. Birkhäuser Advanced Texts, Birkhäuser, Boston, 2001.

[105] H. Grosse, C. Klimčík and P. Prešnajder, Topologically nontrivial field configurations in noncommutative geometry. Commun. Math. Phys. 178 (1996), 507-526.

[106] A. Grossmann, G. Loupias and E. M. Stein, An algebra of pseudodifferential operators and quantum mechanics in phase space. Ann. Inst. Fourier (Grenoble) 18 (1968), 343-368.

[107] P. M. Hajac, Strong connections on quantum principal bundles. Commun. Math. Phys. 182 (1996), 579-617.

[108] P. M. Hajac, ed., Quantum Symmetry and Noncommutative Geometry. Forthcoming, 2006.

[109] P. M. Hajac, M. Khalkhali, B. Rangipour and Y. Sommerhäuser, Hopf-cyclic homology and cohomology with coefficients. C. R. Acad. Sci. Paris 338 (2004), 667-672.

[110] P. M. Hajac and S. Majid, Projective module description of the q-monopole. Commun. Math. Phys. 206 (1999), 247-264.

[111] N. Higson, On the $K$-theory proof of the index theorem. In Index Theory and Operator Algebras (ed. by J. Fox and P. Haskell), Contemp. Math. 148, Amer. Math. Soc., Providence, RI, 1993, 67-86.

[112] N. Higson, The local index formula in noncommutative geometry. In Contemporary Developments in Algebraic K-Theory, (ed. by M. Karoubi, A. O. Kuku and C. Pedrini), ICTP, Trieste, 2004, 443-536.

[113] N. Higson and J. Roe, Analytic K-Homology. Oxford University Press, Oxford, 2000.

[114] M. Hilsum and G. Skandalis, Morphismes $K$-orientés d'espaces de feuilles et fonctorialité en théorie de Kasparov. Ann. Sci. cole Norm. Sup. 20 (1987), 325-390.

[115] Y. Homma, A representation of Spin(4) on the eigenspinors of the Dirac operator on $\mathbb{S}^{3}$. Tokyo J. Math. 23 (2000), 453-472. 
[116] B. Iochum, D. Kastler and T. Schücker, On the universal Chamseddine-Connes action I. Details of the action computation. J. Math. Phys. 38 (1997), 4929-4950.

[117] B. Iochum, T. Krajewski and P. Martinetti, Distances in finite spaces from noncommutative geometry. J. Geom. Phys. 37 (2001), 100-125.

[118] B. Iochum and T. Schücker, Yang-Mills-Higgs versus Connes-Lott. Commun. Math. Phys. 178 (1995), 1-26.

[119] B. Iochum, T. Schücker and C. A. Stephan, On a classification of irreducible almost commutative geometries. J. Math. Phys. 45 (2004), 5003-5041.

[120] R. Jackiw, Physical instances of noncommuting coordinates. Nuclear Phys. B (Proc. Suppl.) 108 (2002), 30-36.

[121] R. V. Kadison and J. R. Ringrose, Fundamentals of the Theory of Operator Algebras II. Academic Press, Orlando, FL, 1986.

[122] W. Kalau and M. Walze, Gravity, noncommutative geometry and the Wodzicki residue. J. Geom. Phys. 16 (1995), 327-344.

[123] G. Karrer, Einführung von Spinoren auf Riemannschen Mannigfaltigkeiten. Ann. Acad. Sci. Fennicae Ser. A I Math. 336 (1963), 3-16.

[124] C. Kassel, Le résidu noncommutatif (d'après M. Wodzicki). Séminaire Bourbaki 41ème année, exposé 708; Astérisque 177 (1989), 199-229.

[125] C. Kassel, Quantum Groups. Grad. Texts in Math. 155, Springer-Verlag, Berlin, 1995.

[126] D. Kastler, The $C^{*}$-algebras of a free boson field. Commun. Math. Phys. 1 (1965), 175-214.

[127] D. Kastler, On A. Connes' noncommutative integration theory. Commun. Math. Phys. 85 (1982), 99-120.

[128] D. Kastler, The Dirac operator and Gravitation. Commun. Math. Phys. 166 (1995), 633-643.

[129] D. Kastler, A second look at $U_{q}\left(S l_{2}\right)$ at third root of unity. J. Math. Phys. 43 (2002), 2770-2790.

[130] D. Kastler and T. Schücker, A detailed account of Alain Connes' version of the Standard Model in noncommutative differential geometry IV. Rev. Math. Phys. 8 (1996), 205-228.

[131] A. U. Klimyk and K. Schmüdgen, Quantum Groups and their Representations. Texts Monogr. Phys., Springer-Verlag, Berlin, 1997. 
[132] M. Kontsevich, Deformation quantization of Poisson manifolds. Lett. Math. Phys. 66 (2003), 157-216.

[133] T. Kopf and M. Paschke, A spectral quadruple for de Sitter space. J. Math. Phys. 43 (2002), 818-846.

[134] Y. A. Kordyukov, Noncommutative geometry of foliations. K-Theory (2006), to appear; Preprint math.dg/0504095, Ufa, 2005.

[135] U. Krähmer, Dirac operators on quantum flag manifolds. Lett. Math. Phys. 67 (2004), $49-59$.

[136] T. Krajewski, Classification of finite spectral triples. J. Geom. Phys. 28 (1998), 1-30.

[137] E. C. Lance, Hilbert $C^{*}$-modules. London Math. Soc. Lecture Note Ser. 210, Cambridge University Press, Cambridge, 1995.

[138] G. Landi, An Introduction to Noncommutative Spaces and their Geometries. Lecture Notes in Phys. New Ser. m Monogr. 51, Springer-Verlag, Berlin, 1997.

[139] G. Landi, F. Lizzi and R. J. Szabo, String geometry and the noncommutative torus. Commun. Math. Phys. 206 (1999), 603-637.

[140] G. Landi, C. Pagani and C. Reina, A Hopf bundle over a quantum four-sphere from the symplectic group. Commun. Math. Phys. 263 (2006), 65-88.

[141] G. Landi and C. Rovelli, General relativity in terms of Dirac eigenvalues. Phys. Rev. Lett. 78 (1997), 3051-3054.

[142] G. Landi and C. Rovelli, Gravity from Dirac eigenvalues. Mod. Phys. Lett. A 13 (1998), 479-494.

[143] G. Landi and W. D. van Suijlekom, Principal fibrations from noncommutative spheres. Commun. Math. Phys. 260 (2005), 203-225.

[144] G. Landi and W. D. van Suijlekom, Noncommutative instantons from twisted conformal symmetries. Preprint math.qa/0601554, Trieste, 2006.

[145] N. P. Landsman, Strict deformation quantization of a particle in external gravitational and Yang-Mills fields. J. Geom. Phys. 12 (1993), 93-132.

[146] N. P. Landsman, Mathematical Topics between Classical and Quantum Mechanics. Springer Monogr. Math., Springer-Verlag, Berlin, 1998.

[147] N. P. Landsman, Lie groupoid $C^{*}$-algebras and Weyl quantization. Commun. Math. Phys. 206 (1999), 367-381. 
[148] N. P. Landsman, Lie Groupoids and Lie algebroids in physics and noncommutative geometry. J. Geom. Phys. 56 (2006), 24-54.

[149] H. B. Lawson, Jr. and M.-L. Michelsohn, Spin Geometry. Princeton University Press, Princeton, NJ, 1989.

[150] S. Lazzarini and T. Schücker, A farewell to unimodularity. Phys. Lett. B 510 (2001), $277-284$.

[151] H. Li, Strong Morita equivalence of higher-dimensional noncommutative tori. J. Reine Angew. Math. 576 (2004), 167-180.

[152] H. Li, $\theta$-Deformations as compact quantum metric spaces. Commun. Math. Phys. 256 (2005), 213-238.

[153] M. Li and Y.-S. Wu, eds., Physics in Noncommutative World I: Field Theories. Rinton Press, Paramus, NJ, 2002.

[154] F. Lizzi and R. J. Szabo, Duality symmetries and noncommutative geometry of string spacetimes. Commun. Math. Phys. 197 (1998), 667-712.

[155] J.-L. Loday, Cyclic Homology. Second edition, Grundlehren Math. Wiss. 301, SpringerVerlag, Berlin, 1998.

[156] S. Lord, A. A. Sedaev and F. A. Sukochev, Dixmier traces as singular symmetric functionals and applications to measurable operators. J. Funct. Anal. 224 (2005), 72106.

[157] J. Madore, An Introduction to Noncommutative Differential Geometry and its Physical Applications. Second edition, Cambridge University Press, Cambridge, 1999.

[158] M. Marcolli, Arithmetic Noncommutative Geometry. University Lecture Series 36, Amer. Math. Soc., Providence, RI, 2005.

[159] C. P. Martín, J. M. Gracia-Bondía and J. C. Várilly, The Standard Model as a noncommutative geometry: the low energy regime. Phys. Reports 294 (1998), 363-406.

[160] J. A. Mignaco, C. Sigaud, A. R. da Silva and F. J. Vanhecke, The Connes-Lott program on the sphere. Rev. Math. Phys. 9 (1997), 689-718.

[161] B. Monthubert and F. Pierrot, Indice analytique et groupoïdes de Lie. C. R. Acad. Sci. Paris 325 (1997), 193-198.

[162] J. E. Moyal, Quantum mechanics as a statistical theory. Proc. Cambridge Philos. Soc. 45 (1949), 99-124.

[163] J. von Neumann, Die Eindeutigkeit der Schrödingerschen Operatoren. Math. Ann. 104 (1931), 570-578. 
[164] E. T. Newman and R. Penrose, Note on the Bondi-Metzner-Sachs group. J. Math. Phys. 7 (1966), 863-870.

[165] M. Paschke and A. Sitarz, Discrete spectral triples and their symmetries. J. Math. Phys. 39 (1998), 6191-6205.

[166] M. Paschke and R. Verch, Locally covariant quantum field theory over spectral geometries. Class. Quant. Grav. 21 (2004), 5299-5316.

[167] D. Pask and A. Rennie, The noncommutative geometry of graph $C^{*}$-algebras I: The index theorem. J. Funct. Anal. 233 (2006), 92-134.

[168] A. L. T. Paterson, Groupoids, Inverse Semigroups and their Operator Algebras. Progress in Mathematics 170, Birkhäuser, Boston, 1999.

[169] S. Paycha and S. Rosenberg, Conformal anomalies via canonical traces. Preprint math.dg/0508290, Clermont-Ferrand, 2005.

[170] M. V. Pimsner and D. Voiculescu, Imbedding the irrational rotation $C^{*}$-algebra into an AF-algebra. J. Operator Theory 4 (1980), 201-210.

[171] R. J. Plymen, Strong Morita equivalence, spinors and symplectic spinors. J. Operator Theory 16 (1986), 305-324.

[172] R. Ponge, Noncommutative residue, conformal invariants and lower dimensional volumes in Riemannian geometry. Preprint math.dg/0604176, MPI, Bonn, 2006.

[173] S. C. Power, Simplicity of $C^{*}$-algebras of minimal dynamical systems. J. London Math. Soc. 18 (1978), 534-538.

[174] P. Prešnajder, The origin of the chiral anomaly and noncommutative geometry. $J$. Math. Phys. 41 (2000), 2789-2804.

[175] I. Raeburn and D. P. Williams, Morita Equivalence and Continuous-Trace $C^{*}$-algebras. Mathematical Surveys and Monographs 60, Amer. Math. Soc., Providence, RI, 1998.

[176] J. Renault, A Groupoid Approach to $C^{*}$-Algebras. Lecture Notes in Math. 793, Springer-Verlag, Berlin, 1980.

[177] A. Rennie, Smoothness and locality for nonunital spectral triples. K-Theory 28 (2003), $127-165$.

[178] A. Rennie, Summability for nonunital spectral triples. K-Theory 31 (2004), 71-100.

[179] M. A. Rieffel, $C^{*}$-algebras associated with irrational rotations. Pacific. J. Math. 93 (1981), 415-429. 
[180] M. A. Rieffel, Von Neumann algebras associated with pairs of lattices in Lie groups. Math. Ann. 257 (1981), 403-418.

[181] M. A. Rieffel, Morita equivalence for operator algebras. In Operator algebras and applications, Part 1 (ed. by Richard V. Kadison), Proc. Symp. Pure Math. 38, Amer. Math. Soc., Providence, RI, 1982, 285-298.

[182] M. A. Rieffel, The cancellation theorem for projective modules over irrational rotation $C^{*}$-algebras. Proc. London Math. Soc. 47 (1983), 285-302.

[183] M. A. Rieffel, Deformation Quantization for Actions of $\mathbb{R}^{d}$. Mem. Amer. Math. Soc. 506, Amer. Math. Soc., Providence, RI, 1993.

[184] M. A. Rieffel, Compact quantum groups associated with toral subgroups. In Representation Theory of Groups and Algebras (ed. by J. Adams et al.), Contemp. Math. 145, Amer. Math. Soc., Providence, RI, 1993, 465-491.

[185] M. A. Rieffel, Metrics on state spaces. Doc. Math. 4 (1999), 559-600.

[186] M. A. Rieffel, Compact quantum metric spaces. In Operator Algebras, Quantization, and Noncommutative Geometry (ed. by R. S. Doran and R. V. Kadison), Contemp. Math. 365, Amer. Math. Soc., Providence, RI, 2004, 315-330.

[187] M. A. Rieffel and A. Schwarz, Morita equivalence of multidimensional noncommutative tori. Int. J. Math. 10 (1999), 289-299.

[188] P. L. Robinson and J. H. Rawnsley, The metaplectic representation, $M p^{c}$ structures and geometric quantization. Mem. Amer. Math. Soc. 410, Amer. Math. Soc., Providence, RI, 1989.

[189] M. Rørdam, F. Larsen and N. Laustsen, An Introduction to K-Theory for $C^{*}$-Algebras. London Mathematical Society Student Texts 49, Cambridge University Press, Cambridge, 2000.

[190] J. Rosenberg, Algebraic K-Theory and its Applications. Grad. Texts in Math. 147, Springer-Verlag, Berlin, 1994.

[191] H. Schröder, On the definition of geometric Dirac operators. Preprint math.dg/ 0005239, Dortmund, 2000.

[192] T. Schücker, Forces from Connes' geometry. In Topology and Geometry in Physics (ed. by E. Bick and F. D. Steffen), Lecture Notes in Phys. 659, Springer-Verlag, Berlin, 2005, 285-350.

[193] T. Schücker, Krajewski diagrams and spin lifts, hep-th/0501181, CPT, Luminy, 2005.

[194] T. Schücker and J.-M. Zylinski, Connes' model building kit. J. Geom. Phys. 16 (1995), $207-236$. 
[195] L. Schwartz, Théorie des Distributions. Hermann, Paris, 1966.

[196] R. T. Seeley, Complex powers of an elliptic operator. In Singular integrals (ed. by A. P. Caldern), Proc. Symp. Pure Math. 10, Amer. Math. Soc., Providence, RI, 1967, 288-307.

[197] I. E. Segal, A noncommutative extension of abstract integration. Ann. of Math. 57 (1953), 401-457.

[198] N. Seiberg and E. Witten, String theory and noncommutative geometry. J. High Energy Phys. 9909 (1999), 032.

[199] A. Sitarz, Rieffel's deformation quantization and isospectral deformations. Int. J. Theor. Phys. 40 (2001), 1693-1696.

[200] A. Sitarz, Quasi-Dirac operators on the sphere. Preprint math-ph/0602030, Düsseldorf, 2006.

[201] H. S. Snyder, Quantized space-time. Phys. Rev. 71 (1947), 38-41.

[202] E. M. Stein, Harmonic Analysis: Real Variable Methods, Orthogonality and Oscillatory Integrals. Princeton University Press, Princeton, NJ, 1994.

[203] A. Strohmaier, On noncommutative and semi-Riemannian geometry. J. Geom. Phys. 56 (2006), 175-195.

[204] W. D. van Suijlekom, L. Da̧browski, G. Landi, A. Sitarz and J. C. Várilly, The local index formula for $\mathrm{SU}_{q}(2)$. K-Theory (2006), to appear.

[205] R. G. Swan, Vector bundles and projective modules. Trans. Amer. Math. Soc. 105 (1962), 264-277.

[206] R. J. Szabo, Quantum field theory on noncommutative spaces. Phys. Reports 378 (2003), 207-299.

[207] M. Takesaki, Tomita's Theory of Modular Hilbert Algebras. Lecture Notes in Maths. 128, Springer-Verlag, Berlin, 1970.

[208] M. E. Taylor, Partial Differential Equations. Volume 2, Appl. Math. Sci. 116, SpringerVerlag, New York, 1996.

[209] W. J. Ugalde, Operadores de Dirac en fibrados de base esférica. M. Sc. thesis, Universidad de Costa Rica, San José, 1996.

[210] W. J. Ugalde, A construction of critical GJMS operators using Wodzicki's residue. Commun. Math. Phys. 261 (2006), 771-788. 
[211] I. Vancea, Euclidean supergravity in terms of Dirac eigenvalues. Phys. Rev. D 58 (1998), 045005.

[212] J. C. Várilly, Quantum symmetry groups of noncommutative spheres. Commun. Math. Phys. 221 (2001), 511-523.

[213] J. C. Várilly and J. M. Gracia-Bondía, Algebras of distributions suitable for phasespace quantum mechanics II. Topologies on the Moyal algebra. J. Math. Phys. 29 (1988), 880-887.

[214] J. C. Várilly and J. M. Gracia-Bondía, The Moyal representation for spin. Ann. Physics (NY) 190 (1989), 107-148.

[215] J. C. Várilly and J. M. Gracia-Bondía, Connes' noncommutative differential geometry and the Standard Model. J. Geom. Phys. 12 (1993), 223-301.

[216] J. C. Várilly and J. M. Gracia-Bondía, On the ultraviolet behaviour of quantum fields over noncommutative manifolds. Int. J. Mod. Phys. A 14 (1999), 1305-1323.

[217] D. V. Vassilevich, Heat kernel expansion: user's manual. Phys. Reports 388 (2003), 279-360.

[218] M. Vergne, Geometric quantization and equivariant cohomology. In ECM: Proceedings of the First European Congress of Mathematics (ed. by A. Joseph, F. Mignot, F. Murat, B. Prum and R. Rentschler), Progress in Mathematics 119, Birkhäuser, Basel, 1994, 249-295.

[219] M. Walze, Nichtkommutative Geometrie und Gravitation. Ph. D. thesis, Johannes Gutenberg Universität, Mainz, 1996.

[220] Y. Wang, Differential forms and the Wodzicki residue for manifolds with boundary. J. Geom. Phys. 56 (2006), 731-753.

[221] N. E. Wegge-Olsen, K-theory and $C^{*}$-algebras, A Friendly Approach. Oxford University Press, Oxford, 1993.

[222] A. Weinstein, Noncommutative geometry and geometric quantization. In Symplectic Geometry and Mathematical Physics (ed. by P. Donato, C. Duval, J. Elhadad and G. M. Tuynman), Progress in Mathematics 99, Birkhuser Boston, Boston, MA, 1991, 446-461.

[223] M. Wodzicki, Noncommutative residue. Chapter I: Fundamentals. In K-theory, Arithmetic and Geometry (ed. by Yu. I. Manin), Lecture Notes in Math. 1289, SpringerVerlag, Berlin, 1987, 320-399.

[224] R. Wulkenhaar, Field theories on deformed spaces. J. Geom. Phys. 56 (2006), 108-141. 